October 4, 2007

\title{
Low energy proton-proton scattering in effective field theory
}

\author{
Shung-ichi Ando ${ }^{1}$, Jae Won Shin, Chang Ho Hyun, and Seung Woo Hong \\ Department of Physics, Sungkyunkwan University, Suwon 440-746, Korea
}

Low energy proton-proton scattering is studied in pionless effective field theory. Employing the dimensional regularization and $\overline{\mathrm{MS}}$ and power divergence subtraction schemes for loop calculation, we calculate the scattering amplitude in ${ }^{1} S_{0}$ channel up to nextto-next-to leading order and fix low-energy constants that appear in the amplitude by effective range parameters. We study regularization scheme and scale dependence in separation of Coulomb interaction from the scattering length and effective range for the $S$-wave proton-proton scattering.

PACS(s): 11.10.Gh, 13.75.Cs.

\footnotetext{
${ }^{1}$ mailto:sando@color.skku.ac.kr
} 


\section{Introduction}

Effective field theories (EFTs), which provide us a systematic perturbative scheme and a model-independent calculation method, have become a popular method to study hadronic reactions with and without external probes at low and intermediate energies. (See, e.g., Refs. [1, 2, 3, 4, 5] for reviews.) At very low energies, the Coulomb interaction becomes essential for the study of reactions involving charged particles. The first consideration of the Coulomb interaction in a pionless EFT was done by Kong and Ravndal $(\mathrm{KR})$ for low energy $S$-wave proton-proton $(p p)$ scattering $[6,7]$. They calculated the $p p$ scattering amplitude up to next-to leading order (NLO). For loop calculations, they employed dimensional regularization with minimum subtraction (MS) scheme and so called power divergence subtraction (PDS) scheme suggested by Kaplan, Savage and Wise [8, 9]. Then KR estimated a scattering length $a(\mu)$ for the $p p$ scattering after separating off the Coulomb correction where $\mu$ is the scale for dimensional regularization. The leading order (LO) result of $a(\mu)$ was almost infinite at $\mu=m_{\pi}$ where $m_{\pi}$ is the pion mass [6]. In addition, the LO $a(\mu)$ was highly dependent on the value of $\mu$. Including the NLO correction, they obtained $a\left(\mu=m_{\pi}\right)=-29.9 \mathrm{fm}$ [7] which is comparable to the value of the scattering length $a_{n p}$ in the $n p$ channel, $a_{n p}=-23.748 \pm 0.009 \mathrm{fm}^{2}$.

The value of $a(\mu)$ deduced after separating the Coulomb and strong interactions is particularly important in the study of isospin breaking effects in $S$-wave $N N$ interaction $[11,12]$. The accurate value of $a_{n p}$ is well known as quoted above, while the values of the scattering length in the $n n$ channel $\left(a_{n n}\right)$ and in the $p p$ channel $\left(a_{p p}\right)$ still have considerable uncertainties.

There exists no direct $n n$ scattering experiment because of the lack of free neutron target. The values of $a_{n n}$ have been deduced from the experimental data of $\pi^{-} d \rightarrow n n \gamma$ and $n d \rightarrow n n p$ reactions. Recent publications suggest $a_{n n}=-18.50 \pm 0.05($ stat. $) \pm$ 0.44 (syst.) \pm 0.30 (th.) fm from the $\pi^{-} d \rightarrow n n \gamma$ process [13] and $a_{n n}=-18.7 \pm 0.6$ $\mathrm{fm}[14],-16.06 \pm 0.35 \mathrm{fm}[15]$ and $-16.5 \pm 0.9 \mathrm{fm}[16]$ from the $n d \rightarrow n n p$ process. As seen, the values of $a_{n n}$ have significant errors compared to that of $a_{n p}$, and the center values do not seem to converge yet. ${ }^{3}$

For the $p p$ channel, a very accurate value of the scattering length $a_{C}=-7.828 \pm 0.008$ $\mathrm{fm}[19]$ and $a_{C}=-7.8149 \pm 0.0029 \mathrm{fm}[20]$ are available from the low energy $p p$ scattering data. It contains however contributions from both strong and electromagnetic interactions, and thus we need to disentangle the strong interaction from the electromagnetic interaction. It was pointed out in potential model calculations that there is a considerable model dependence in deducing the value of the strong scattering length $a_{p p}$ from $a_{C}[19,21]$. Some literature shows $a_{p p}=-17.1 \pm 0.2 \mathrm{fm}[19]$, while a heavy-baryon chiral perturbation theory results in $a_{p p}=-17.51 \sim-16.96 \mathrm{fm}[22]$ with uncertainties slightly larger than those from the potential models.

In this work, we employ the pionless EFT [23] including the Coulomb interaction between two protons $[6,7]$ and calculate the $p p$ scattering amplitude with the strong

\footnotetext{
${ }^{2}$ See, e.g., Table VIII in Ref. [10].

${ }^{3}$ Recently, there were proposals to determine the value of $a_{n n}$ more precisely by employing a formalism of EFT, from the $\pi^{-} d \rightarrow n n \gamma$ reaction[17] and neutron-neutron fusion, $n n \rightarrow d e^{-} \bar{\nu}_{e}[18]$.
} 
$N N$ interactions up to next-to-next-to leading order (NNLO). Our main motivation of this study is to see how the value of strong scattering length $a\left(\mu=m_{\pi}\right)=-29.9 \mathrm{fm}$ obtained by KR from NLO calculations may be improved by the inclusion of a higher order correction. We find that the NNLO corrections turn out to be quite small but there is a considerable dependence of the scattering length $a(\mu)$ on the renormalization schemes and the scale parameter $\mu$.

This paper is organized as follows. In Sec. 2 we briefly review the effective range formalism for the $p p$ scattering. In Sec. 3 the pionless strong effective Lagrangian up to NNLO is introduced. In Sec. 4 we calculate the $S$-wave $p p$ scattering amplitude up to NNLO. In Sec. 5, we discuss regularization method and renormalization schemes employed in this work. We renormalize low energy constants (LECs) that appear in the strong $N N$ interaction up to NNLO by effective range parameters employing MS-bar $(\overline{\mathrm{MS}})$ and PDS schemes and obtain numerical results for the strong scattering length $a(\mu)$ and strong effective range $r(\mu)$. Discussion and conclusions are given in Sec. 6. In Appendix A we show detailed expressions of the amplitudes in NNLO. Detailed calculations of the loop functions employing the dimensional regularization and $\overline{\mathrm{MS}}$ and PDS schemes are given in Appendix B.

\section{Proton-proton scattering in effective range theory}

The amplitude of the $p p$ scattering can be decomposed as [24]

$$
T=T_{C}+T_{S C}
$$

where $T_{C}$ is the pure Coulomb part and $T_{S C}$ is the "modified" strong amplitude whose $S$-wave channel we calculate up to NNLO in pionless EFT below.

The incoming and outgoing scattering states $\left|\Psi_{\vec{p}}^{( \pm)}\right\rangle$with the potential $\hat{V}=\hat{V}_{C}+\hat{V}_{S}$ where $\hat{V}_{C}$ and $\hat{V}_{S}$ are the Coulomb and strong potentials, respectively, are represented in terms of the Coulomb states $\left|\psi_{\vec{p}}^{( \pm)}\right\rangle$as

$$
\left|\Psi_{\vec{p}}^{( \pm)}\right\rangle=\sum_{n=0}^{\infty}\left(\hat{G}_{C}^{( \pm)} \hat{V}_{S}\right)^{n}\left|\psi_{\vec{p}}^{( \pm)}\right\rangle,
$$

where $\hat{G}_{C}^{( \pm)}$is the incoming and outgoing Green's function

$$
\hat{G}_{C}^{( \pm)}(E)=\frac{1}{E-\hat{H}_{0}-\hat{V}_{C} \pm i \epsilon} .
$$

Here $\hat{H}_{0}=\hat{p}^{2} / M$ is the free Hamiltonian of two protons and $V_{C}=e^{2} /(4 \pi r)$ is the repulsive Coulomb potential. The Coulomb state $\left|\psi_{\vec{p}}^{( \pm)}\right\rangle$is obtained by solving the Schrödinger equation $(\hat{H}-E)\left|\psi_{\vec{p}}^{( \pm)}\right\rangle=0$ with $\hat{H}=\hat{H}_{0}+\hat{V}_{C}$ and thus one has

$$
\left|\psi_{\vec{p}}^{( \pm)}\right\rangle=\left[1+\hat{G}_{C}^{( \pm)} \hat{V}_{C}\right]|\vec{p}\rangle
$$


where $|\vec{p}\rangle$ is the free wave state. The normalization of $\left|\psi_{\vec{p}}^{( \pm)}\right\rangle$is such that $\left\langle\psi_{\vec{p}}^{( \pm)} \mid \psi_{\vec{q}}^{( \pm)}\right\rangle=$ $(2 \pi)^{3} \delta^{(3)}(\vec{p}-\vec{q})$. The amplitude $T_{S C}$ is thus obtained by

$$
T_{S C}\left(\vec{p}^{\prime}, \vec{p}\right)=\sum_{n=0}^{\infty}\left\langle\psi_{\vec{p}^{\prime}}^{(-)}\left|\hat{V}_{S}\left(\hat{G}_{C}^{(+)} \hat{V}_{S}\right)^{n}\right| \psi_{\vec{p}}^{(+)}\right\rangle
$$

For $l=0$ state one has the amplitude

$$
T_{S C}^{l=0}=-\frac{4 \pi}{M} \frac{e^{2 i \sigma_{0}}}{p \cot \delta_{0}-i p}
$$

where $\sigma_{l}$ is the Coulomb phase shift $\sigma_{l}=\arg \Gamma(1+l+i \eta)$ with $\eta=\alpha M /(2 p)$. In the effective range expansion with the Coulomb interaction, the modified strong phase shift $\delta_{l}$ for $l=0$ in low energy $p p$ scattering is represented by effective range parameters [25]:

$$
C_{\eta}^{2} p \cot \delta_{0}+\alpha M h(\eta)=-\frac{1}{a_{C}}+\frac{1}{2} r_{0} p^{2}-P r_{0}^{3} p^{4}+\cdots,
$$

where $C_{\eta}^{2}=2 \pi \eta /\left(e^{2 \pi \eta}-1\right)$ and

$$
h(\eta)=\operatorname{Re} \psi(i \eta)-\ln \eta .
$$

$\psi$-function is the logarithmic derivative of the Gamma function and $\operatorname{Re} \psi(i \eta)=\eta^{2} \sum_{\nu=1}^{\infty} \frac{1}{\nu\left(\nu^{2}+\eta^{2}\right)}-$ $C_{E} ; C_{E}$ is the Euler's constant, $C_{E}=0.577215 \cdots$. Effective range parameters $a_{C}, r_{0}, P$ are modified scattering length, effective range, effective volume, respectively.

\section{Effective Lagrangian}

Pionless effective Lagrangian for strong $S$-wave $N N$ interaction up to NNLO reads [23, 26]

$$
\begin{aligned}
\mathcal{L}= & N^{\dagger}\left(i D_{0}+\frac{\vec{D}^{2}}{2 m_{N}}\right) N-C_{0}\left[N^{T} P_{a}^{\left({ }^{1} S_{0}\right)} N\right]^{\dagger} N^{T} P_{a}^{\left({ }^{1} S_{0}\right)} N \\
& +\frac{1}{2} C_{2}\left[N^{T} P_{a}^{\left(1 S_{0}\right)} \stackrel{\leftrightarrow}{D}^{2} N\right]^{\dagger} N^{T} P_{a}^{\left({ }^{1} S_{0}\right)} N+\text { h.c. } \\
& \left.-\frac{1}{2} C_{4}\left(N^{T} P_{a}^{(1} S_{0}\right) \stackrel{\leftrightarrow}{D}^{2} N\right)^{\dagger} N^{T} P_{a}^{\left({ }^{1} S_{0}\right)} \stackrel{\leftrightarrow}{D}^{2} N \\
& \left.-\frac{1}{4} \tilde{C}_{4}\left[\left(N^{T} P_{a}^{(1} S_{0}\right) \stackrel{\leftrightarrow}{D}^{4} N\right)^{\dagger} N^{T} P_{a}^{\left(1 S_{0}\right)} N+\text { h.c. }\right],
\end{aligned}
$$

where $D_{\mu}$ is the covariant derivative, $\stackrel{\leftrightarrow}{D}=\frac{1}{2}(\vec{D}-\overleftarrow{D})$, and $P_{a}^{\left({ }^{1} S_{0}\right)}$ is a projection operator for the two-nucleon ${ }^{1} S_{0}$ states, $P_{a}^{\left({ }^{1} S_{0}\right)}=\frac{1}{\sqrt{8}} \sigma_{2} \tau_{2} \tau_{a}$. Note that we retain two low energy constants, $C_{4}$ and $\tilde{C}_{4}$, in NNLO.

The strong $N N$ potential is expanded in terms of small momentum as

$$
\hat{V}_{S}=\hat{V}_{0}+\hat{V}_{2}+\hat{V}_{4}+\cdots
$$


where $\hat{V}_{0}, \hat{V}_{2}, \hat{V}_{4}$ are LO, NLO, NNLO potential, respectively, and the matrix elements of them are obtained from the Lagrangian in Eq. (9) as

$$
\begin{aligned}
\left\langle\vec{q}\left|\hat{V}_{0}\right| \vec{k}\right\rangle & =C_{0}, \\
\left\langle\vec{q}\left|\hat{V}_{2}\right| \vec{k}\right\rangle & =\frac{1}{2} C_{2}\left(\vec{q}^{2}+\vec{k}^{2}\right), \\
\left\langle\vec{q}\left|\hat{V}_{4}\right| \vec{k}\right\rangle & =\frac{1}{2} C_{4} \vec{q}^{2} \vec{k}^{2}+\frac{1}{4} \tilde{C}_{4}\left(\vec{q}^{4}+\vec{k}^{4}\right),
\end{aligned}
$$

where $|\vec{q}\rangle$ and $|\vec{k}\rangle$ are the intermediate free two-nucleon outgoing and incoming states, respectively: $2 \vec{q}$ and $2 \vec{k}$ are the relative momenta for the two protons.

In this work we employ the standard counting rules of the strong $N N$ interaction with the PDS scheme in Refs. [7, 8]. (We will discuss the PDS scheme in detail later.) For the strong potential, the LO term $C_{0}$ is counted as $Q^{-1}$ order, where $Q$ denotes the small expansion parameter, and is summed up to an infinite order. The NLO $\left(C_{2}\right)$ and NNLO $\left(C_{4}, \tilde{C}_{4}\right)$ terms are counted as $Q^{2}$ and $Q^{4}$, respectively, and expanded perturbatively. ${ }^{4}$ We treat the Coulomb interaction non-perturbatively using the Green's function $G_{C}^{( \pm)}$in Eq. (3). We do not include higher order QED corrections such as the vacuum polarization effects reported in Refs. [27].

\section{Amplitudes}

The amplitude $T_{S C}^{l=0}$ for the $S$-wave $p p$ scattering can be written as

$$
T_{S C}^{l=0}=T_{S C}^{(0)}+T_{S C}^{(2)}+T_{S C}^{(4)}+\cdots,
$$

where $T_{S C}^{(0)}, T_{S C}^{(2)}, T_{S C}^{(4)}$ are LO, NLO, NNLO amplitudes, respectively. By inserting the strong LO potential $\hat{V}_{0}$ in Eq. (11) into the amplitude $T_{S C}$ in Eq. (5), we obtain the LO amplitude $T_{S C}^{(0)}$ in terms of loop functions $\psi_{0}$ and $J_{0}$ :

$$
T_{S C}^{(0)}=\sum_{n=0}^{\infty}\left\langle\psi_{\vec{p}^{\prime}}^{(-)}\left|\hat{V}_{0}\left(\hat{G}_{C}^{(+)} \hat{V}_{0}\right)^{n}\right| \psi_{\vec{p}}^{(+)}\right\rangle=\frac{C_{0} \psi_{0}^{2}(p)}{1-C_{0} J_{0}(p)},
$$

where

$$
\begin{aligned}
\psi_{0}(p) & =\int \frac{d^{3} \vec{k}}{(2 \pi)^{3}} \psi_{\vec{p}}^{(+)}(\vec{k})=\int \frac{d^{3} \vec{k}}{(2 \pi)^{3}} \psi_{\vec{p}}^{(-) *}(\vec{k}) \\
J_{0}(p) & =\int \frac{d^{3} \vec{k}^{\prime}}{(2 \pi)^{3}} \frac{d^{3} \vec{q}}{(2 \pi)^{3}}\left\langle\vec{q}\left|\hat{G}_{C}^{(+)}\right| \vec{k}^{\prime}\right\rangle .
\end{aligned}
$$

Detailed calculations for the functions $\psi_{0}$ and $J_{0}$ are given in Appendix B. $T_{S C}^{(0)}$ is summation of the LO strong potential $\hat{V}_{0}$, that is, the $C_{0}$ terms summed up to the infinite order.

\footnotetext{
${ }^{4}$ Note that by changing the LECs $C_{4}$ and $\tilde{C}_{4}$ in another linear combination, e.g., $C_{4}=C_{4}^{\prime}+\tilde{C}_{4}^{\prime}$ and $\tilde{C}_{4}=C_{4}^{\prime}-\tilde{C}_{4}^{\prime}$, one can easily see that the term proportional to $\tilde{C}_{4}^{\prime}$ in Eq. (13) vanishes when $|\vec{q}|=|\vec{k}|$. The $\tilde{C}_{4}^{\prime}$ term, so called off-shell term, is redundant and vanishes when the external legs of the potential go on mass-shell.
} 
(a)

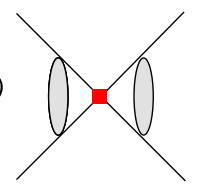

(b)

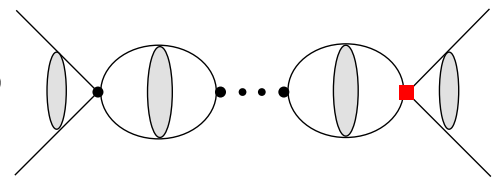

(c)

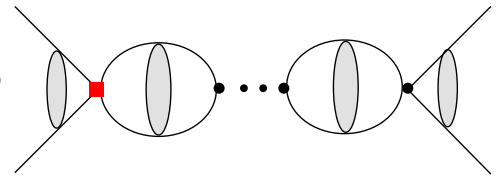

(d)

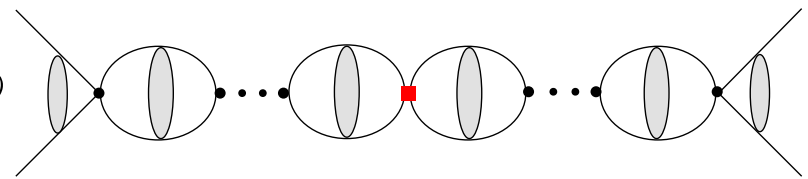

Figure 1: NLO diagrams for the $S$-wave $p p$ scattering. Gray blobs denote the twoproton Coulomb Green's function $G_{C}^{(+)}$, and two nucleon contact vertices denote the strong potential: the (black) circle and the (red) square represent $\mathrm{LO}\left(C_{0}\right)$ and $\mathrm{NLO}\left(C_{2}\right)$ vertices, respectively. Small double dots stand for the summation of $C_{0}$ terms up to the infinite order.

At NLO we have four diagrams shown in Fig. $1 .{ }^{5}$ They are proportional to $C_{2}$ coming from $V_{2}$, whereas the $C_{0}$ terms are summed up to the infinite order. The NLO amplitude is written in terms of the loop functions $\psi_{0}, \psi_{2}, J_{0}$ and $J_{2}$ as

$$
T_{S C}^{(2, a-d)}=\frac{C_{2} \psi_{0}}{\left(1-C_{0} J_{0}\right)^{2}}\left[\psi_{2}+C_{0}\left(\psi_{0} J_{2}-\psi_{2} J_{0}\right)\right],
$$

with

$$
\begin{aligned}
\psi_{2}(p) & =\int \frac{d^{3} \vec{k}}{(2 \pi)^{3}} \vec{k}^{2} \psi_{\vec{p}}^{(+)}(\vec{k})=\int \frac{d^{3} \vec{k}}{(2 \pi)^{3}} \vec{k}^{2} \psi_{\vec{p}}^{(-) *}(\vec{k}), \\
J_{2}(p) & =\int \frac{d^{3} \vec{q}}{(2 \pi)^{3}} \frac{d^{3} \vec{q}^{\prime}}{(2 \pi)^{3}} \vec{q}^{2}\left\langle\vec{q}^{\prime}\left|\hat{G}_{C}^{(+)}\right| \vec{q}\right\rangle=\int \frac{d^{3} \vec{q}}{(2 \pi)^{3}} \frac{d^{3} \vec{q}^{\prime}}{(2 \pi)^{3}}\left\langle\vec{q}^{\prime}\left|\hat{G}_{C}^{(+)}\right| \vec{q}\right\rangle \vec{q}^{2} .
\end{aligned}
$$

Details for $\psi_{2}$ and $J_{2}$ are given in Appendix B. The NLO amplitude $T_{S C}^{(2)}$ consists of one $C_{2}$ and a summation of the $C_{0}$ terms up to the infinite order. These LO and NLO amplitudes have already been obtained by KR in Ref. [7].

At NNLO we have three sets of diagrams shown in Figs. 2, 3, and 4. From the first and

\footnotetext{
${ }^{5}$ Figures were prepared using the program JaxoDraw [28] provided by L. Theussl.
} 
(a)

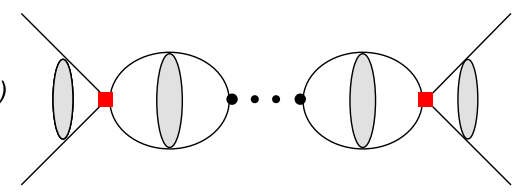

(b)

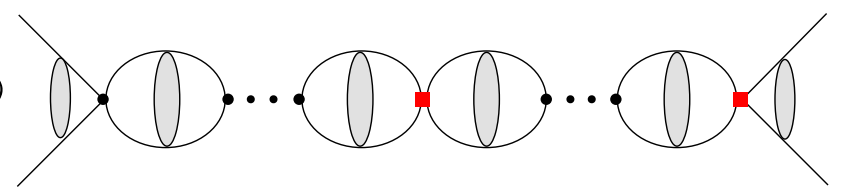

(c)

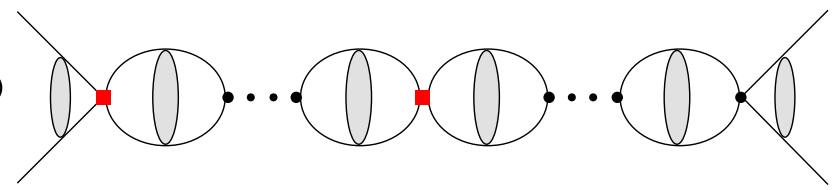

(d)

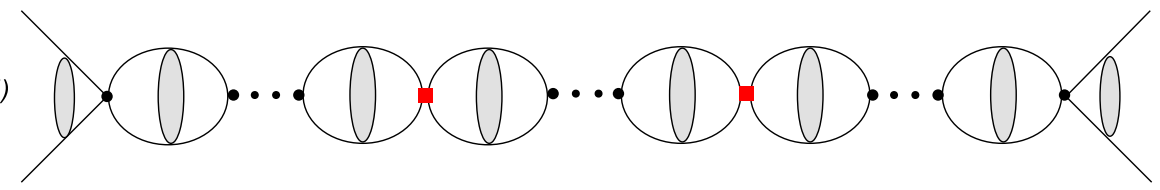

Figure 2: Set 1 of NNLO diagrams. See the caption of Fig. 1 for details.

second sets of diagrams shown in Figs. 2 and 3, respectively, we see two NLO corrections to the amplitude and thus the NNLO amplitudes obtained from the first and second sets of diagrams in Figs. 2 and 3 are proportional to $C_{2}^{2}$. The NNLO amplitudes corresponding to the diagrams in Fig. 2 can be written in terms of the functions $\psi_{0}, \psi_{2}, J_{0}$ and $J_{2}$, whereas to express the amplitudes for the diagrams in Fig. 3 we need a new function $J_{22}$ given below. In the third set of diagrams shown in Fig. 4, we have one NNLO correction to the amplitude and the NNLO amplitudes for the diagrams in Fig. 4 are proportional to $C_{4}$ or $\tilde{C}_{4}$. Explicit expressions of the NNLO amplitude from each of the diagrams are given in terms of $\psi_{i}$ with $i=0,2,4$ and $J_{j}$ with $j=0,2,22,4$ in Appendix A.

Summing up the amplitudes obtained from the diagrams (a) to (h) in Figs. 2 and 3 we have

$$
\begin{aligned}
T_{S C}^{(4, a-h)}= & \frac{C_{2}^{2}}{4\left(1-C_{0} J_{0}\right)^{3}}\left\{\psi_{0}^{2} J_{22}\left(1-C_{0} J_{0}\right)+\psi_{2}^{2} J_{0}\left(1-C_{0} J_{0}\right)^{2}\right. \\
& \left.+2 \psi_{0} \psi_{2} J_{2}\left(1-C_{0}^{2} J_{0}^{2}\right)+\psi_{0}^{2} J_{2}\left(C_{0} J_{2}\right)\left(3+C_{0} J_{0}\right)\right\}
\end{aligned}
$$

where

$$
J_{22}=\int \frac{d^{3} \vec{q}}{(2 \pi)^{3}} \frac{d^{3} \vec{q}^{\prime}}{(2 \pi)^{3}} \vec{q}^{2}\left\langle\vec{q}^{\prime}\left|\hat{G}_{C}^{(+)}\right| \vec{q}\right\rangle \vec{q}^{2},
$$

whose details are given in Appendix B. 
(e)

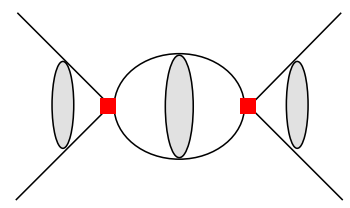

(f)

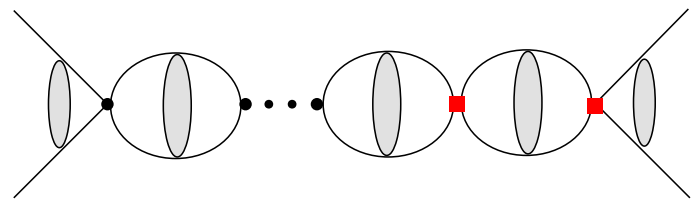

(g)

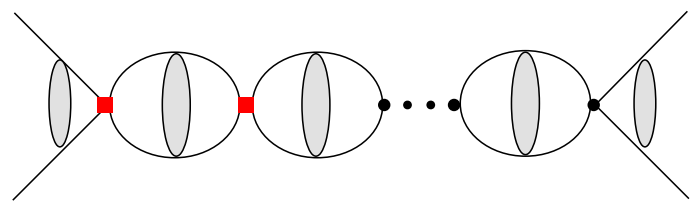

(h)

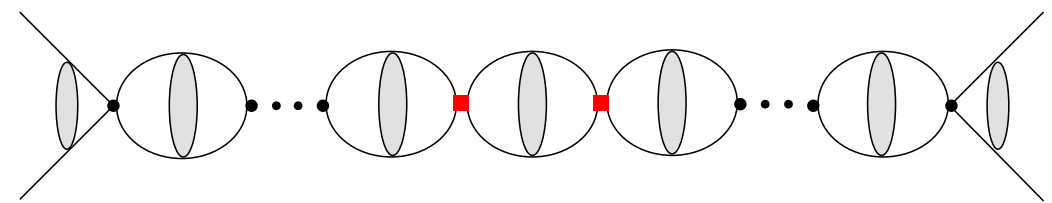

Figure 3: Set 2 of NNLO diagrams. See the caption of Fig. 1 for details.

Summing up the amplitudes for the diagrams (i) to (l) in Fig. 4 gives us

$$
\begin{aligned}
T_{S C}^{(4, i-l)}= & \frac{1}{2} \frac{C_{4}}{\left(1-C_{0} J_{0}\right)^{2}}\left[\psi_{2}^{2}\left(1-C_{0} J_{0}\right)^{2}+2 \psi_{0} \psi_{2} C_{0} J_{2}\left(1-C_{0} J_{0}\right)+\psi_{0}^{2} C_{0}^{2} J_{2}^{2}\right] \\
& +\frac{1}{2} \frac{\tilde{C}_{4}}{\left(1-C_{0} J_{0}\right)^{2}}\left[\psi_{4}+C_{0}\left(\psi_{0} J_{4}-\psi_{4} J_{0}\right)\right] \psi_{0},
\end{aligned}
$$

where

$$
\begin{aligned}
\psi_{4} & =\int \frac{d^{3} \vec{k}}{(2 \pi)^{3}} \psi_{\vec{p}}^{(-) *}(\vec{k}) \vec{k}^{4}=\int \frac{d^{3} \vec{k}}{(2 \pi)^{3}} \vec{k}^{4} \psi_{\vec{p}}^{(+)}(\vec{k}), \\
J_{4} & =\int \frac{d^{3} \vec{q}}{(2 \pi)^{3}} \frac{d^{3} \vec{q}^{\prime}}{(2 \pi)^{3}} \vec{q}^{4}\left\langle\vec{q}^{\prime}\left|\hat{G}_{C}^{(+)}\right| \vec{q}\right\rangle=\int \frac{d^{3} \vec{q}}{(2 \pi)^{3}} \frac{d^{3} \vec{q}^{\prime}}{(2 \pi)^{3}}\left\langle\vec{q}^{\prime}\left|\hat{G}_{C}^{(+)}\right| \vec{q}\right\rangle \vec{q}^{4} .
\end{aligned}
$$

Calculations of $\psi_{4}$ and $J_{4}$ are given in Appendix B.

\section{Regularization method and renormalization schemes}

In the calculation of the loop functions $J_{0}, J_{2}, J_{22}$ and $J_{4}$ in Eqs. (17), (20), (22), (25), we encounter infinities and employ the dimensional regularization. We also employ the PDS scheme, suggested by Kaplan, Savage and Wise [8, 9], in which one subtracts the poles in $d=3$ as well as those in $d=4$ space-time dimensions so that one obtains an expected perturbation series in the expansion of the $N N$ potential in Eq. (10) with a 

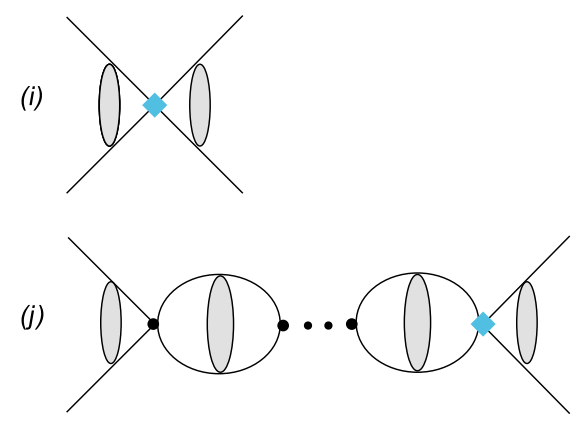

$(k)$
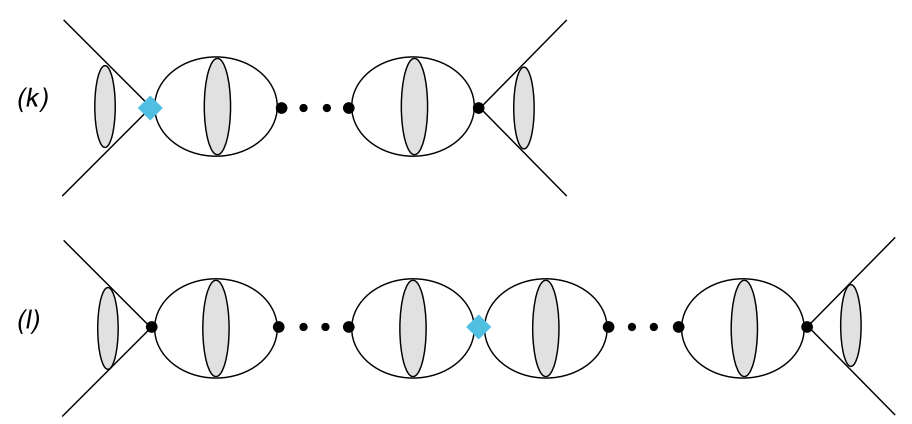

Figure 4: Set 3 of NNLO diagrams. Two-proton contact vertices represented by (blue) diamonds denote strong NNLO potential $V_{4}$. See the caption of Fig. 1 for details.

given scale $\mu$ of the theory. We may check the convergence radius, e.g., for the $C_{2}$ term (relative to the $C_{0}$ term) in Eq. (10) and have $\Lambda_{20}(\mu) \equiv \sqrt{C_{0}(\mu) / C_{2}(\mu)}=147$ (30.6) $\mathrm{MeV}$ with (without) the PDS terms at $\mu=m_{\pi}$. Thus a formal convergence of the perturbative series of the $N N$ potential in Eq. (10) is improved thanks to the PDS term, and the theory would be valid up to $p \sim \Lambda_{20} \simeq 140 \mathrm{MeV}$, which is the large scale we assumed in the pionless theory.

The loop functions can be decomposed into a finite term and an infinite one, e.g. $J_{0}=J_{0}^{f i n}+J_{0}^{d i v}$ with $J_{0}^{f i n}=-\frac{\alpha M^{2}}{4 \pi} H(\eta)$ (the definition of the $H(\eta)$ function is given in Appendix B) and

$$
J_{0}^{d i v}=-\frac{M}{4 \pi} \mu+\frac{\alpha M^{2}}{8 \pi}\left[\frac{1}{\epsilon}-3 C_{E}+2+\ln \left(\frac{\pi \mu^{2}}{\alpha^{2} M^{2}}\right)\right],
$$

where $J_{0}^{d i v}$ is calculated by the dimensional regularization in $d=4-2 \epsilon$ dimensions and the PDS scheme. The first term proportional to the scale $\mu$ in the r.h.s. of Eq. (26) is the PDS term and $C_{E}$ is the Euler's constant mentioned earlier. The scattering amplitudes should be identical after renormalization even if another renormalization scheme such as off-shell momentum subtraction scheme discussed in Refs. [26, 29] is employed. However, $a(\mu)$ and $r(\mu)$ do depend on the renormalization schemes along with the value of the renormalization scale $\mu$. So, to be consistent with $K R$, we calculate all the loop functions $J_{i}$ with $i=0,2,22,4$ and the wavefunctions $\psi_{j}$ with $j=0,2,4$ by using the dimensional 
regularization and the PDS scheme in Appendix B.

The $S$-wave $p p$ scattering amplitude in terms of the effective range parameters is given by

$$
T_{S C}^{l=0}=-\frac{4 \pi}{M} \frac{C_{\eta}^{2} e^{2 i \sigma_{0}}}{-\alpha M H(\eta)-\frac{1}{a_{C}}+\frac{1}{2} r_{0} p^{2}-P r_{0}^{3} p^{4}+\cdots},
$$

and thus one has

$$
\begin{aligned}
& -\frac{1}{a_{C}}+\frac{1}{2} r_{0} p^{2}-\operatorname{Pr}_{0}^{3} p^{4}+\cdots=\alpha M H(\eta)-\frac{4 \pi}{M} C_{\eta}^{2} e^{2 i \sigma_{0}} \frac{1}{T_{S C}^{l=0}} \\
& =\alpha M H(\eta)-\frac{4 \pi}{M} \frac{\psi_{0}^{2}}{T_{S C}^{(0)}}\left[1-\frac{T_{S C}^{(2)}}{T_{S C}^{(0)}}-\frac{T_{S C}^{(4)}}{T_{S C}^{(0)}}+\left(\frac{T_{S C}^{(2)}}{T_{S C}^{(0)}}\right)^{2}+\cdots\right] .
\end{aligned}
$$

Comparing the coefficients of the terms proportional to $p^{0}, p^{2}$ and $p^{4}$ in both sides of Eq. (28), we have

$$
\begin{aligned}
-\frac{1}{a_{C}}= & -\frac{4 \pi}{M}\left\{\frac{1}{C_{0}}-J_{0}^{d i v}+\frac{C_{2}}{C_{0}^{2}}\left[\alpha M \mu+\frac{1}{2}(\alpha M)^{2}+C_{0} \frac{\pi M}{48}(\alpha M)^{2} \mu\right]\right. \\
& \left.-\left(\frac{1}{2} \frac{C_{4}}{C_{0}^{2}}-\frac{C_{2}^{2}}{C_{0}^{3}}\right)(\alpha M)^{2} \mu^{2}\right\}+\mathcal{O}\left(\alpha^{3}\right), \\
+\frac{1}{2} r_{0}= & \frac{4 \pi}{M}\left[\frac{C_{2}}{C_{0}^{2}}-2\left(\frac{1}{2} \frac{C_{4}}{C_{0}^{2}}+\frac{1}{3} \frac{\tilde{C}_{4}}{C_{0}^{2}}-\frac{C_{2}^{2}}{C_{0}^{3}}\right)(\alpha M) \mu\right]+\mathcal{O}\left(\alpha^{2}\right), \\
-P r_{0}^{3}= & \frac{4 \pi}{M}\left(\frac{1}{2} \frac{C_{4}}{C_{0}^{2}}+\frac{1}{2} \frac{\tilde{C}_{4}}{C_{0}^{2}}-\frac{C_{2}^{2}}{C_{0}^{3}}\right),
\end{aligned}
$$

where we have expanded the r.h.s. of Eqs. (29) and (30) in the order of the fine structure constant $\alpha$ and neglected the $\alpha^{3}\left(\alpha^{2}\right)$ and higher order terms in Eq. (29) (Eq.(30)). With three effective range parameters, we cannot determine the four LECs uniquely. There are some arguments which can constrain the values of $C_{4}$ and $\tilde{C}_{4}$. The $C_{4}$ contribution in Eq. (29) is of the order of $\mu^{2}$, and thus the first $\tilde{C}_{4}$ contribution term is of the lower order of $\mu$ than the $C_{4}$ term. ${ }^{6}$ For this reason, the $\tilde{C}_{4}$ term is treated as an order higher than the $C_{4}$ one [23], and consequently the $\tilde{C}_{4}$ term does not appear (at NNLO) in Eq. (29). The other argument is based on the offshell-ness of a term proportional to $C_{4}-\tilde{C}_{4}[26]{ }^{7}$ In this case, the term proportional to $C_{4}-\tilde{C}_{4}$ is redundant and thus can be removed by assuming $C_{4}=\tilde{C}_{4}$. Because both arguments seem to have some grounds, to check the dependency of the results on the values of $C_{4}$ and $\tilde{C}_{4}$ we consider the three cases: 1) $\tilde{C}_{4}=0$ (Ref. [23]), 2) $C_{4}=\tilde{C}_{4}$ (Ref. [26]), and 3) $C_{4}=0$.

In Eq. (29) there is the $J_{0}^{d i v}$ term explicitly given in Eq. (26). In the MS scheme used by $\mathrm{KR}[6,7]$ one subtracts the infinite term $\frac{\alpha M^{2}}{8 \pi} \frac{1}{\epsilon}$ from the $J_{0}^{d i v}$. One can use another

\footnotetext{
${ }^{6}$ Note that $\mu$ is regarded as a large scale, i.e., $\mu=m_{\pi}$.

${ }^{7}$ See the footnote 4 .
} 
scheme called $\overline{\mathrm{MS}}$ scheme, in which finite terms are subtracted together with the infinite term so that $\frac{\alpha M^{2}}{8 \pi}\left[\frac{1}{\epsilon}-C_{E}+\ln (4 \pi)\right]$ is subtracted. Then we have

$$
J_{0}^{\overline{M S}}=-\frac{M}{4 \pi} \mu+\frac{\alpha M^{2}}{4 \pi}\left[\ln \left(\frac{\mu}{2 \alpha M}\right)+1-C_{E}\right] .
$$

This leads to a significant subtraction scheme dependence in the scattering length $a(\mu)$.

\section{Numerical results}

We may define the strong scattering length and the effective range, respectively, in the zeroth order of $\alpha$ as [7]

$$
\frac{1}{a(\mu)}=\frac{4 \pi}{M} \frac{1}{C_{0}(\mu)}+\mu, \quad \frac{1}{2} r(\mu)=\frac{4 \pi}{M} \frac{C_{2}(\mu)}{C_{0}^{2}(\mu)} .
$$

Inserting the expressions of $a(\mu)$ and $r(\mu)$ in Eqs. (33) into Eqs. (29) and (30), we have

$$
\begin{aligned}
& \frac{1}{a(\mu)}=\left[\frac{1}{a(\mu)}\right]_{L O}+\left[\frac{1}{a(\mu)}\right]_{N L O}+\left[\frac{1}{a(\mu)}\right]_{N N L O}, \\
& r(\mu)=r_{0}-(\alpha M)\left[D_{3} P r_{0}^{3} \mu+D_{4} \frac{r_{0}^{2} \mu}{\frac{1}{a_{C}}-\mu}\right]
\end{aligned}
$$

where

$$
\begin{aligned}
{\left[\frac{1}{a(\mu)}\right]_{L O} } & =\frac{1}{a_{C}}+\alpha M\left[\ln \left(\frac{\mu}{2 \alpha M}\right)+1-C_{E}\right], \\
{\left[\frac{1}{a(\mu)}\right]_{N L O} } & =-\frac{1}{2} \alpha M r_{0} \mu-(\alpha M)^{2}\left[\frac{1}{4} r_{0}+\frac{\pi^{2}}{12} \frac{r_{0} \mu}{\frac{1}{a_{C}}-\mu}\right], \\
{\left[\frac{1}{a(\mu)}\right]_{N N L O} } & =(\alpha M)^{2}\left[D_{1} P r_{0}^{3} \mu^{2}-\frac{D_{2} r_{0} \mu}{12} \frac{r_{0} \mu}{\frac{1}{a_{C}}-\mu}\right],
\end{aligned}
$$

and the term linear in $\alpha M$ in Eq. (35) is the NNLO correction to $r(\mu)$. We have three set of coefficients, $X_{x(=1,2,3)}=\left\{D_{1}, D_{2}, D_{3}, D_{4}\right\}$, because of the additional constraints imposed on the LECs $C_{4}$ and $\tilde{C}_{4}$ mentioned before Eq. (32). $X_{1}=\{1,0,4,0\}$ corresponds to the case 1) $\tilde{C}_{4}=0, X_{2}=\{7 / 6,1,10 / 3,1 / 6\}$ corresponds to the case 2) $\tilde{C}_{4}=C_{4}$, and $X_{3}=\{4 / 3,-10,8 / 3,1 / 3\}$ to the case 3$) C_{4}=0$. We use the values of effective range parameters,

$$
a_{C}=-7.82 \mathrm{fm}, \quad r_{0}=2.78 \mathrm{fm}, \quad P \simeq 0.022 .
$$

We can also have explicit expressions for the LECs $C_{0}(\mu), C_{2}(\mu), C_{4}(\mu)$ and $\tilde{C}_{4}(\mu)$ from Eqs. (34), (35) and (31) with the constraints for $C_{4}$ and $\tilde{C}_{4}$.

In Fig. 5 we plot our result of the strong scattering length $a(\mu)$ as a function of the scale parameter $\mu$. In the left panel, we plot three curves for the strong scattering length 

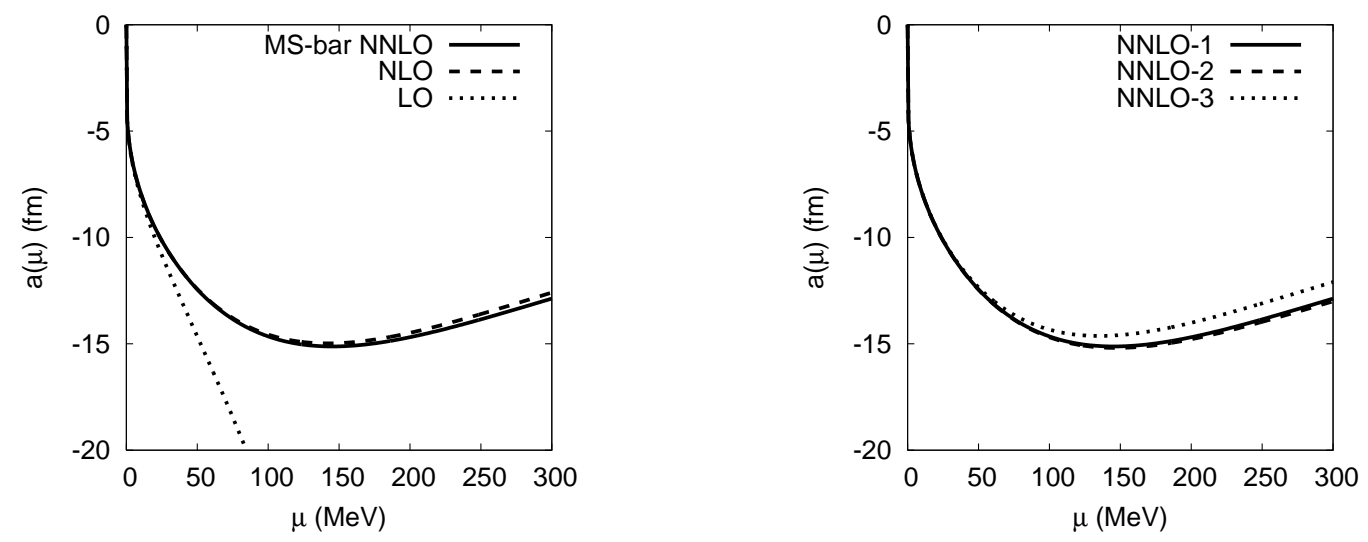

Figure 5: Strong scattering length $a(\mu)[\mathrm{fm}]$ in functions of the scale parameter $\mu[\mathrm{MeV}]$. In the left panel, $a(\mu)$ is plotted by the dotted curve, the dashed curve, and the full curve, respectively, for up to LO, NLO, and NNLO. In the right panel, $a(\mu)$ calculated up to NNLO are plotted for the three different constraints for $C_{4}$ and $\tilde{C}_{4}$, which are explained in the text.

$a(\mu)$ up to LO, NLO, and NNLO with the constraint $\tilde{C}_{4}=0$ (the case 1 ). We find that the NLO correction significantly improves the estimation of $a(\mu)$, as shown by KR.

If one looks into the details more closely, however, there is a quantitative difference in the results of LO and NLO between the MS and $\overline{\mathrm{MS}}$ schemes. The value of the LO scattering length $a_{L O}(\mu)$ at $\mu=m_{\pi}$ in the $\overline{\mathrm{MS}}$ scheme, which is obtained from Eq. (36), is $a_{L O}^{\overline{M S}}\left(\mu=m_{\pi}\right)=-30.72 \mathrm{fm}$. The LO contributions to $a(\mu)$ can be divided into three terms; $1 / a_{C}$, the term proportional to a log function and the remaining ones proportional to $\alpha M$. Evaluating each contribution, we obtain $1 / a_{C}=-0.1279, \alpha M \ln \left(\frac{m_{\pi}}{2 \alpha M}\right)=0.0807$ and $\alpha M\left(1-C_{E}\right)=0.0147$ in units of $\mathrm{fm}^{-1}$ in the $\overline{\mathrm{MS}}$ scheme. There is a strong cancellation between $1 / a_{C}$ and the $\log$ term which has the order of $\alpha M$. Consequently $1 / a\left(\mu=m_{\pi}\right)$ becomes a small value, making its inverse large. In the case of the MS scheme, the cancellation is stronger, having the $\log$ term $\alpha M \ln \left(\frac{\pi m_{\pi}^{2}}{\alpha^{2} M^{2}}\right)=0.1247$ and $\alpha M\left(-3 C_{E}+\right.$ $2) / 2=0.0047$ in units of $\mathrm{fm}^{-1}$. The cancellation of $1 / a_{C}$ and the terms proportional to $\alpha M$ makes the value of $1 / a\left(\mu=m_{\pi}\right)$ two orders of magnitude smaller than $1 / a_{C}$. As a result, one gets an unrealistically huge scattering length, $a_{L O}^{M S}\left(\mu=m_{\pi}\right)=738.62 \mathrm{fm}$. The strong dependence on the renormalization schemes of the LO contribution to $a(\mu)$ makes the EFT result somehow arbitrary.

The NLO contribution, Eq. (37) can be divided into terms linear in $\alpha M$ and those proportional to $(\alpha M)^{2}$. The term linear in $\alpha M$ is comparable in magnitude with the LO contribution because of the cancellation in LO, as discussed above. More precisely, we have $1 / a_{L O}^{\overline{M S}}=-0.0325$ and $-\alpha M r_{0} \mu / 2=-0.0343$ in units of $\mathrm{fm}^{-1}$. On the other hand, the numerical value of the contribution proportional to $(\alpha M)^{2}$ is 0.0015 in units of $\mathrm{fm}^{-1}$, which is about $5 \%$ of the terms linear in $\alpha M$. 


\begin{tabular}{c|cccc}
\hline & NLO & NNLO-1 & NNLO-2 & NNLO-3 \\
\hline$a(\mu)$ & -14.98 & -15.11 & -15.18 & -14.62 \\
$r(\mu)$ & - & 2.73 & 2.78 & 2.82 \\
\hline
\end{tabular}

Table 1: Numerical estimations (in units of $\mathrm{fm}$ ) of scattering length $a(\mu)$ and effective range $r(\mu)$ up to NLO and NNLO without Coulomb effect at $\mu=140 \mathrm{MeV}$.

The NNLO contribution is very small, as can be seen from the left and right panels in Fig. 5 and Table 1. The reason can be easily found from the expressions for the NNLO terms in Eq. (38). These terms are proportional to $(\alpha M)^{2}$. We observed in NLO that the $(\alpha M)^{2}$ term is smaller than the $\alpha M$ order term by an order of magnitude. The magnitude of $(\alpha M)^{2}$ terms in NNLO ranges from about $20 \%$ to $300 \%$ of $(\alpha M)^{2}$ terms in NLO, depending on the choice of the assumptions on $C_{4}$ and $\tilde{C}_{4}$. Consequently, the NNLO correction to $1 / a(\mu)$ is about $1 \sim 6 \%$ of the contributions up to NLO, depending on the constraints of $C_{4}$ and $\tilde{C}_{4}$.

In Table 1 we show the estimated values of the strong scattering length $a(\mu)$ and effective range $r(\mu)$ at $\mu=m_{\pi} \cdot{ }^{8}$ The NNLO term itself varies by an order of magnitude depending on the choice of the constraints on $C_{4}$ and $\tilde{C}_{4}$. However, as discussed in a previous paragraph, its contribution to $a(\mu)$ is suppressed due to a higher order of $\alpha M$ factor. As a result, the different choice of the constraints on $C_{4}$ and $\tilde{C}_{4}$ affects little the final result, only a few percents at most. The first correction to $r(\mu)$ appears at NNLO and is linear in $\alpha M$, whereas the NLO correction to $1 / a(\mu)$ does in the $\alpha M$ order. Contrary to the case of $1 / a(\mu)$ where the $\alpha M$ correction plays a crucial role, the $\alpha M$ contribution to $r(\mu)$ amounts to only about $2 \%$ of $r_{0}$. Though the $\alpha M$ order corrections to $1 / a(\mu)$ and $r(\mu)$ are of the same order of magnitude, the $(\alpha M)^{0}$ order contribution to $1 / a_{C}$ is smaller than that of $r_{0}$ by an order of magnitude. Consequently, we have very contrasting behavior of $a(\mu)$ and $r(\mu)$.

Thus our results of the strong $p p$ scattering length and effective range up to NNLO, which are estimated by employing the dimensional regularization and the $\overline{\mathrm{MS}}$ and PDS schemes at $\mu=m_{\pi}$, can be summarized as

$$
\begin{aligned}
& a\left(\mu=m_{\pi}\right)=-14.9 \pm 0.3 \mathrm{fm}, \\
& r\left(\mu=m_{\pi}\right)=2.78 \pm 0.05 \mathrm{fm},
\end{aligned}
$$

where the error-bars are estimated by the uncertainties due to the constraints on $C_{4}$ and $\tilde{C}_{4}$, which could play a similar role to the model dependence in deducing the values of the strong scattering length $a_{p p}$ and effective range $r_{0, p p}$ in the potential model calculations.

\section{Discussion and conclusions}

\footnotetext{
${ }^{8}$ We find a minimum point for $a(\mu)$ at $\mu \simeq 2 / r_{0} \simeq 142 \mathrm{MeV}$, which is very close to the pion mass, $\mu=m_{\pi}$.
} 
In this work, we calculated the $S$-wave $p p$ scattering amplitude up to NNLO in the framework of the pionless EFT. The loop functions were calculated by using the dimensional regularization with the $\overline{\mathrm{MS}}$ and PDS schemes. After fixing the LECs by using the effective range parameters, we estimated the strong scattering length $a(\mu)$ and the strong effective range $r(\mu)$ as functions of $\mu$. The LO contributions to $1 / a(\mu)$ are composed of $1 / a_{C}$ and the terms depending on $\alpha M$ arising from the loop diagrams. The smallness of $1 / a_{C}$ makes it comparable in magnitude to the $\alpha M$ terms in the same order. Due to the opposite signs of $1 / a_{C}$ and the $\alpha M$ terms, furthermore, there is a strong cancellation among them and thus it makes the LO result for $1 / a(\mu)$ suppressed and sensitive to the renormalization schemes. The NLO correction, expanded in powers of $\alpha M$, begins with the linear order of $\alpha M$. The linear $\alpha M$ order correction to $a(\mu)$ is of the same order of magnitude as the $\alpha M$ terms in LO, and thus makes the NLO contribution crucial in both of the MS and $\overline{\mathrm{MS}}$ schemes. The higher $\alpha M$ order terms in NLO, e.g., the terms proportional to $(\alpha M)^{2}$ are suppressed to a few percents of the leading contribution, so they can be regarded as a perturbative corrections to both $a(\mu)$ and $r(\mu)$. The NNLO terms give us only a fairly minor correction to the results up to NLO. The reason is partly attributed to the additional order counting of the NNLO terms in powers of $\alpha M$ : The $\alpha M$ order corrections in NNLO begin with $(\alpha M)^{2}$. Similar to the $(\alpha M)^{2}$ contribution in NLO, the terms in NNLO produces small corrections to the results. In conclusion, we can say that our investigation reveals both bright and shadowy aspects of studying the strong $p p$ scattering length in EFT. Convergence from NLO to NNLO is satisfactory, but the LO and NLO results are significantly dependent on the renormalization schemes.

Though the quantities of the strong scattering length and effective range from the $p p$ scattering could be regarded as physical quantities, it is unlikely that they can be determined unambiguously without the subtraction scheme and renormalization scale dependence within the present framework of EFT. Similar arguments can be found in Refs. [30, 31]. Nevertheless, the strong $p p$ scattering length and effective range are important ingredients for better understanding of the isospin nature of the $N N$ interaction. The problem of the strong $p p$ scattering length may have to be approached at various levels, from "first principle calculations" like lattice QCD to more complex systems in which $a(\mu)$ (or equivalently $C_{0}(\mu)$ ) plays non-trivial roles.

\section{Acknowledgments}

We thank Yoonbai Kim for a useful comment on our work. S.A. thanks F. Ravndal for communications. S.A. is supported by Korean Research Foundation and The Korean Federation of Science and Technology Societies Grant funded by Korean Government (MOEHRD, Basic Research Promotion Fund): the Brain Pool program (052-1-6) and KRF-2006-311-C00271.

\section{Appendix A: Amplitudes in NNLO}

In this appendix we present expressions of each of the amplitudes in NNLO in terms of functions, $\psi_{0,2,4}$ and $J_{0,2,22,4}$. Detailed calculations of the $\psi$ and $J$ functions are given 
in Appendix B. From the diagram (a) in Fig. 2, we have

$$
\begin{aligned}
T_{S C}^{(4, a)} & =\left\langle\psi_{\vec{p}^{\prime}}^{(-)}\left|\hat{V}_{2} \hat{G}_{C}^{(+)} \sum_{n=0}^{\infty}\left(\hat{V}_{0} \hat{G}_{C}^{(+)}\right)^{n} \hat{V}_{0} G_{C}^{(+)} \hat{V}_{2}\right| \psi_{\vec{p}}^{(+)}\right\rangle \\
& =\frac{C_{0}}{1-C_{0} J_{0}} \int \frac{d^{3} \vec{q}^{\prime}}{(2 \pi)^{3}}\left\langle\psi_{\vec{p}^{\prime}}^{(-)}\left|\hat{V}_{2} \hat{G}_{C}^{(+)}\right| \vec{q}^{\prime}\right\rangle \int \frac{d^{3} \vec{q}}{(2 \pi)^{3}}\left\langle\vec{q}\left|\hat{G}_{C}^{(+)} \hat{V}_{2}\right| \psi_{\vec{p}}^{(+)}\right\rangle \\
& =\frac{1}{4} \frac{C_{0} C_{2}^{2}}{1-C_{0} J_{0}}\left(\psi_{0} J_{2}+\psi_{2} J_{0}\right)^{2} .
\end{aligned}
$$

From the diagrams (b) and (c) in Fig. 2 we have

$$
\begin{aligned}
T_{S C}^{(4, b, c)}= & \left\langle\psi_{\vec{p}^{\prime}}^{(-)}\left|\sum_{n=0}^{\infty}\left(\hat{V}_{0} G_{C}^{(+)}\right)^{n} \hat{V}_{0} \hat{G}_{C}^{(+)} \hat{V}_{2} \hat{G}_{C}^{(+)} \sum_{m=0}^{\infty}\left(\hat{V}_{0} G_{C}^{(+)}\right)^{m} \hat{V}_{0} G_{C}^{(+)} \hat{V}_{2}\right| \psi_{\vec{p}}^{(+)}\right\rangle \\
& +\left\langle\psi_{\vec{p}^{\prime}}^{(-)}\left|\hat{V}_{2} \hat{G}_{C}^{(+)} \sum_{n=0}^{\infty}\left(\hat{V}_{0} G_{C}^{(+)}\right)^{n} \hat{V}_{0} \hat{G}_{C}^{(+)} \hat{V}_{2} \hat{G}_{C}^{(+)} \sum_{m=0}^{\infty}\left(\hat{V}_{0} G_{C}^{(+)}\right)^{m} \hat{V}_{0}\right| \psi_{\vec{p}}^{(+)}\right\rangle \\
= & \frac{C_{0}^{2} \psi_{0}}{\left(1-C_{0} J_{0}\right)^{2}} \int \frac{d^{3} \vec{q}}{(2 \pi)^{3}} \frac{d^{3} \vec{q}^{\prime}}{(2 \pi)^{3}}\left\langle\vec{q}^{\prime}\left|\hat{G}_{C}^{(+)} \hat{V}_{2} \hat{G}_{C}^{(+)}\right| \vec{q}\right\rangle \\
& \times \int \frac{d^{3} \vec{k}}{(2 \pi)^{3}}\left[\left\langle\vec{k}\left|\hat{G}_{C}^{(+)} \hat{V}_{2}\right| \psi_{\vec{p}}^{(+)}\right\rangle+\left\langle\psi_{\vec{p}^{\prime}}^{(-)}\left|\hat{V}_{2} \hat{G}_{C}^{(+)}\right| \vec{k}\right\rangle\right] \\
= & \frac{C_{0}^{2} C_{2}^{2} \psi_{0} J_{0} J_{2}}{\left(1-C_{0} J_{0}\right)^{2}}\left(\psi_{0} J_{2}+\psi_{2} J_{0}\right) .
\end{aligned}
$$

From the diagram (d) in Fig. 2, we have

$$
\begin{aligned}
T_{S C}^{(4, d)} & =\left\langle\psi_{\vec{p}^{\prime}}^{(-)}\left|\sum_{l=0}^{\infty}\left(\hat{V}_{0} \hat{G}_{C}^{(+)}\right)^{l} \hat{V}_{0} \hat{G}_{C}^{(+)} \hat{V}_{2} \hat{G}_{C}^{(+)} \sum_{m=0}^{\infty}\left(\hat{V}_{0} \hat{G}_{C}^{(+)}\right)^{m} \hat{V}_{0} \hat{G}_{C}^{(+)} \hat{V}_{2} \hat{G}_{C}^{(+)} \sum_{n=0}^{\infty}\left(\hat{V}_{0} \hat{G}_{C}^{(+)}\right)^{n} \hat{V}_{0}\right| \psi_{\vec{p}}^{(+)}\right\rangle \\
& =\frac{C_{0}^{3} \psi_{0}^{2}}{\left(1-C_{0} J_{0}\right)^{3}}\left[\int \frac{d^{3} \vec{q}^{\prime}}{(2 \pi)^{3}} \frac{d^{3} \vec{q}}{(2 \pi)^{3}}\left\langle\vec{q}^{\prime}\left|\hat{G}_{C}^{(+)} \hat{V}_{2} \hat{G}_{C}^{(+)}\right| \vec{q}\right\rangle\right]^{2}=\frac{C_{0}^{3} C_{2}^{2} \psi_{0}^{2}}{\left(1-C_{0} J_{0}\right)^{3}} J_{0}^{2} J_{2}^{2} .
\end{aligned}
$$

From the diagram (e) in Fig. 3 we have

$$
T_{S C}^{(4, e)}=\left\langle\psi_{\vec{p}^{\prime}}^{(-)}\left|\vec{V}_{2} \hat{G}_{C}^{(+)} \hat{V}_{2}\right| \psi_{\vec{p}}^{(+)}\right\rangle=\frac{C_{2}^{2}}{4}\left(\psi_{0}^{2} J_{22}+\psi_{2}^{2} J_{0}+2 \psi_{0} \psi_{2} J_{2}\right) .
$$

From the diagrams (f) and (g) in Fig. 3 we have

$$
\begin{aligned}
T_{S C}^{(4, f, g)}= & \left\langle\psi_{\vec{p}^{\prime}}^{(-)}\left|\sum_{n=0}^{\infty}\left(\hat{V}_{0} \hat{G}_{C}^{(+)}\right)^{n} \hat{V}_{0} \hat{G}_{C}^{(+)} \hat{V}_{2} \hat{G}_{C}^{(+)} \hat{V}_{2}\right| \psi_{\vec{p}}^{(+)}\right\rangle \\
& +\left\langle\psi_{\vec{p}^{\prime}}^{(-)}\left|\hat{V}_{2} \hat{G}_{C}^{(+)} \hat{V}_{2} \hat{G}_{C}^{(+)} \sum_{n=0}^{\infty}\left(\hat{V}_{0} \hat{G}_{C}^{(+)}\right)^{n} \hat{V}_{0}\right| \psi_{\vec{p}}^{(+)}\right\rangle \\
= & \frac{1}{2} \frac{C_{0} C_{2}^{2} \psi_{0}}{1-C_{0} J_{0}}\left(\psi_{0} J_{2}^{2}+2 \psi_{2} J_{0} J_{2}+\psi_{0} J_{0} J_{22}\right) .
\end{aligned}
$$


From the diagram (h) in Fig. 3 we have

$$
\begin{aligned}
T_{S C}^{(4, h)} & =\left\langle\psi_{\vec{p}^{\prime}}^{(-)}\left|\sum_{m=0}^{\infty}\left(\hat{V}_{0} \hat{G}_{C}^{(+)}\right)^{m} \hat{V}_{0} \hat{G}_{C}^{(+)} \hat{V}_{2} \hat{G}_{C}^{(+)} \hat{V}_{2} \hat{G}_{C}^{(+)} \sum_{n=0}^{\infty}\left(\hat{V}_{0} \hat{G}_{C}^{(+)}\right)^{n} \hat{V}_{0}\right| \psi_{\vec{p}}^{(+)}\right\rangle \\
& =\frac{1}{4} \frac{C_{0}^{2} C_{2}^{2} \psi_{0}^{2}}{\left(1-C_{0} J_{0}\right)^{2}}\left(3 J_{0} J_{2}^{2}+J_{0}^{2} J_{22}\right) .
\end{aligned}
$$

From the diagram (i) in Fig. 4 we have

$$
T_{S C}^{(4, i)}=\left\langle\psi_{\vec{p}}^{(-)}\left|\hat{V}_{4}\right| \psi_{\vec{p}}^{(+)}\right\rangle=\frac{1}{2} C_{4} \psi_{2}^{2}+\frac{1}{2} \tilde{C}_{4} \psi_{0} \psi_{4} .
$$

From the diagrams (j) and (k) in Fig. 4 we have

$$
\begin{aligned}
T_{S C}^{(4, j, k)} & =\left\langle\psi_{\vec{p}^{\prime}}^{(-)}\left|\sum_{n=0}^{\infty}\left(\hat{V}_{0} \hat{G}_{C}^{(+)}\right)^{n} \hat{V}_{0} \hat{G}_{C}^{(+)} \hat{V}_{4}\right| \psi_{\vec{p}}^{(+)}\right\rangle+\left\langle\psi_{\vec{p}^{\prime}}^{(-)}\left|\hat{V}_{4} \hat{G}_{C}^{(+)} \sum_{n=0}^{\infty}\left(\hat{V}_{0} \hat{G}_{C}^{(+)}\right)^{n} \hat{V}_{0}\right| \psi_{\vec{p}}^{(+)}\right\rangle \\
& =\frac{1}{2} \frac{C_{0} \psi_{0}}{1-C_{0} J_{0}}\left[2 C_{4} \psi_{2} J_{2}+\tilde{C}_{4}\left(\psi_{0} J_{4}+\psi_{4} J_{0}\right)\right]
\end{aligned}
$$

From the diagram (l) in Fig. 4 we have

$$
\begin{aligned}
T_{S C}^{(4, l)} & =\left\langle\psi_{\vec{p}^{\prime}}^{(-)}\left|\sum_{m=0}^{\infty}\left(\hat{V}_{0} \hat{G}_{C}^{(+)}\right)^{m} \hat{V}_{0} \hat{G}_{C}^{(+)} \hat{V}_{4} \hat{G}_{C}^{(+)} \sum_{n=0}^{\infty}\left(\hat{V}_{0} \hat{G}_{C}^{(+)}\right)^{n} \hat{V}_{0}\right| \psi_{\vec{p}}^{(+)}\right\rangle \\
& =\frac{1}{2} \frac{C_{0}^{2} \psi_{0}^{2}}{\left(1-C_{0} J_{0}\right)^{2}}\left[C_{4} J_{2}^{2}+\tilde{C}_{4} J_{0} J_{4}\right] .
\end{aligned}
$$

\section{Appendix B: Loop functions}

In this appendix, we present $\psi$ functions $\left(\psi_{0}, \psi_{2}, \psi_{4}\right)$ and $J$ functions $\left(J_{0}, J_{2}, J_{22}\right.$, and $J_{4}$ ) employing dimensional regularization and power divergent regularization scheme $[7,8]$. We first show the calculations of the $\psi_{0}, \psi_{2}, \psi_{4}$ functions in Eqs. (16), (19), (24).

1. $\psi_{0}$ : The Fourier transformation of the Coulomb wavefunction $\psi_{\vec{p}}^{( \pm)}(\vec{r})$ is

$$
\psi_{\vec{p}}^{( \pm)}(\vec{k})=\int d^{3} \vec{r} \psi_{\vec{p}}^{( \pm)}(\vec{r}) e^{-i \vec{k} \cdot \vec{r}}
$$

with

$$
\psi_{\vec{p}}^{( \pm)}(\vec{r})=\sum_{l=0}^{\infty}(2 l+1) i^{l} R_{l}^{( \pm)}(p r) P_{l}(\cos \theta),
$$

where $\cos \theta=\hat{p} \cdot \hat{r}$. One has the relation, $\vec{k} \cdot \vec{r}=k r[\cos \theta \cos \hat{\theta}+\sin \theta \sin \hat{\theta} \cos (\phi-\hat{\phi})]$, where $\vec{r}$ and $\vec{k}$ are represented by $(r, \theta, \phi)$ and $(k, \hat{\theta}, \hat{\phi})$, respectively. Now we choose $\hat{\phi}=0$ and then have

$$
\int_{0}^{2 \pi} d \phi e^{-i k r \sin \theta \sin \hat{\theta} \cos \phi}=2 \pi J_{0}(-k r \sin \theta \sin \hat{\theta})
$$


where $J_{n}$ is a Bessel function and we have used the Bessel's first integral, $J_{n}(z)=$ $\frac{1}{2 \pi i^{n}} \int_{0}^{2 \pi} d \phi e^{i z \cos \phi} e^{i n \phi}$. Using the relations,

$$
\int_{0}^{\pi} d \theta \sin \theta P_{l}(\cos \theta) J_{0}(-k r \sin \theta \sin \hat{\theta}) e^{-i k r \cos \theta \cos \hat{\theta}}=i \sqrt{\frac{2 \pi}{-k r}} P_{l}(\cos \theta) J_{l+\frac{1}{2}}(-k r),
$$

$J_{l}(-z)=(-1)^{l} J_{l}(z)$, and $j_{l}(z)=\sqrt{\frac{\pi}{2 z}} J_{l+\frac{1}{2}}(z)$ where Eq. (54) is obtained from Eq. (15) in Ref. [32], we have

$$
\psi_{\vec{p}}^{( \pm)}(\vec{k})=4 \pi \sum_{l=0}^{\infty}(2 l+1) P_{l}(\cos \hat{\theta}) \int_{0}^{\infty} d r r^{2} R_{l}^{( \pm)}(p r) j_{l}(k r)
$$

Now we calculate $\psi_{0}$ by the dimensional regularization. The angular integration will pick up the $l=0$ part of the wavefunction, thus we have

$$
\begin{aligned}
\psi_{0}(p) & =\left(\frac{\mu}{2}\right)^{4-d} \int \frac{d^{d-1} \vec{k}}{(2 \pi)^{d-1}} \psi_{\vec{p}}^{(+)}(\vec{k}) \\
& =4 \pi\left(\frac{\mu}{2}\right)^{4-d} \frac{\Omega_{d-1}}{(2 \pi)^{d-1}} \int_{0}^{\infty} d r r^{2} R_{0}^{(+)}(p r) \int_{0}^{\infty} d k k^{d-2} j_{0}(k r) \\
& =(2 \pi)^{3 / 2}\left(\frac{\mu}{2}\right)^{4-d} \frac{\Omega_{d-1}}{(2 \pi)^{d-1}} \int_{0}^{\infty} d r r^{3-d} R_{0}^{(+)}(p r) \int_{0}^{\infty} d \rho \rho^{d-\frac{5}{2}} J_{\frac{1}{2}}(\rho) .
\end{aligned}
$$

Using the relation $\int_{0}^{\infty} d t t^{\alpha-1} J_{\nu}(t)=\frac{2^{\alpha-1}}{\Gamma\left(\frac{1}{2}(2-\alpha+\nu)\right)} \Gamma\left(\frac{\alpha+\nu}{2}\right)$, we have

$$
\int_{0}^{\infty} d \rho \rho^{d-\frac{5}{2}} J_{\frac{1}{2}}(\rho)=2^{d-\frac{5}{2}} \frac{\Gamma\left(\frac{d-1}{2}\right)}{\Gamma\left(\frac{4-d}{2}\right)} .
$$

Furthermore, from Eq. (6.64) of Ref. [24] we have $R_{0}^{(+)}(p r)=e^{i \sigma_{0}} C_{\eta 1} F_{1}(1+i \eta, 2 ;-2 i p r) e^{i p r}$, where ${ }_{1} F_{1}(a ; b ; z)$ is the confluent hypergeometric function (or Kummer's function of the first kind). Using the relation, $\int_{0}^{\infty} e^{-t} t^{b-1}{ }_{1} F_{1}(a, c ; t z)=\Gamma(b)_{2} F_{1}(a, b, c ; z)$, where ${ }_{2} F_{1}(a, b ; c ; z)$ is the first hypergeometric function, we have

$$
\int_{0}^{\infty} e^{i p r} r^{3-d} F_{1}(1+i \eta, 2,-2 i p r)=\Gamma(4-d)(-i p){ }_{2}^{d-4} F_{1}(1+i \eta, 4-d, 2 ; 2),
$$

and thus

$\psi_{0}=(2 \pi)^{3 / 2}\left(\frac{\mu}{2}\right)^{4-d} \frac{\Omega_{d-1}}{(2 \pi)^{d-1}} e^{i \sigma_{0}} C_{\eta} \Gamma(4-d)(-i p)^{d-4}{ }_{2} F_{1}(1+i \eta, 4-d, 2 ; 2) 2^{d-\frac{5}{2}} \frac{\Gamma\left(\frac{d-1}{2}\right)}{\Gamma\left(\frac{4-d}{2}\right)}$.

There are no poles at $d=3$ and 4 in Eq. (59). Using the relation ${ }_{2} F_{1}(1+i \eta, 0,2 ; 2)=1$ and $\Omega_{d}=2 \pi^{d / 2} / \Gamma(d / 2)$, we have

$$
\psi_{0}=e^{i \sigma_{0}} C_{\eta} .
$$


2. $\psi_{2}$ :

$$
\begin{aligned}
& \psi_{2}(p)=\left(\frac{\mu}{2}\right)^{4-d} \int \frac{d^{d-1} \vec{k}}{(2 \pi)^{d-1}} \psi_{\vec{p}}^{(+)}(\vec{k}) \vec{k}^{2} \\
& =(2 \pi)^{3 / 2}\left(\frac{\mu}{2}\right)^{4-d} \frac{\Omega_{d-1}}{(2 \pi)^{d-1}} \int_{0}^{\infty} d r r^{1-d} R_{0}^{(+)}(p r) \int_{0}^{\infty} d \rho \rho^{d-\frac{1}{2}} J_{\frac{1}{2}}(\rho) \\
& =(2 \pi)^{3 / 2}\left(\frac{\mu}{2}\right)^{4-d} \frac{\Omega_{d-1}}{(2 \pi)^{d-1}} e^{i \sigma_{0}} C_{\eta}(-i p)^{d-2}{ }_{2} F_{1}(1+i \eta, 2-d, 2 ; 2) 2^{d-\frac{3}{2}} \frac{\Gamma\left(\frac{d+1}{2}\right)}{3-d} \frac{\Gamma(4-d)}{\Gamma\left(\frac{4-d}{2}\right)}
\end{aligned}
$$

For $d=4$ we have

$$
\psi_{2}=e^{i \sigma_{0}} C_{\eta}\left(p^{2}-\frac{1}{2} \alpha^{2} M^{2}\right)
$$

where we have used the relation ${ }_{2} F_{1}(1+i \eta,-2,2,2)=\frac{1}{3}-\frac{2}{3} \eta^{2}$. For $d=3$ we have

$$
\psi_{2}^{(d=3)}=-e^{i \sigma_{0}} C_{\eta} \alpha M \mu \frac{1}{3-d}+\cdots,
$$

where we have used the relation ${ }_{2} F_{1}(1+i \eta,-1,2 ; 2)=-i \eta$, and thus we have

$$
\psi_{2}=e^{i \sigma_{0}} C_{\eta}\left[p^{2}-\alpha M \mu-\frac{1}{2}(\alpha M)^{2}\right]
$$

3. $\psi_{4}$ :

$$
\begin{aligned}
\psi_{4} & =\left(\frac{\mu}{2}\right)^{4-d} \int \frac{d^{d-1} \vec{k}}{(2 \pi)^{d-1}} \psi_{\vec{p}}^{(+)}(\vec{k}) \vec{k}^{4} \\
& =(2 \pi)^{3 / 2}\left(\frac{\mu}{2}\right)^{4-d} \frac{\Omega_{d-1}}{(2 \pi)^{d-1}} \int_{0}^{\infty} d r r^{-1-d} R_{0}^{(+)}(p r) \int_{0}^{\infty} d \rho \rho^{d+\frac{3}{2}} J_{\frac{1}{2}}(\rho) \\
& =(2 \pi)^{3 / 2}\left(\frac{\mu}{2}\right)^{4-d} \frac{\Omega_{d-1}}{(2 \pi)^{d-1}} e^{i \sigma_{0}} C_{\eta}(-i p)^{d}{ }_{2} F_{1}(1+i \eta,-d, 2 ; 2) \frac{2^{d+\frac{3}{2}} \Gamma\left(\frac{d+3}{2}\right)}{4(1-d)(3-d)} \frac{\Gamma(4-d)}{\Gamma\left(\frac{4-d}{2}\right)}
\end{aligned}
$$

For $d=4$ we have

$$
\psi_{4}=e^{i \sigma_{0}} C_{\eta}\left(p^{4}-\frac{5}{6} \alpha^{2} M^{2} p^{2}+\frac{1}{24} \alpha^{4} M^{4}\right)
$$

where we have used the relation ${ }_{2} F_{1}(1+i \eta,-4,2 ; 2)=\frac{1}{15}\left(3-10 \eta^{2}+2 \eta^{4}\right)$. For $d=3$ we have

$$
\psi_{4}^{d=3}=-\frac{4}{3} e^{i \sigma_{0}} C_{\eta} \alpha M \mu\left(p^{2}-\frac{1}{8} \alpha^{2} M^{2}\right) \frac{1}{3-d}+\cdots
$$


where we have used the relation ${ }_{2} F_{1}(1+i \eta,-3,2 ; 2)=\frac{i}{3} \eta\left(-2+\eta^{2}\right)$. Thus we have

$$
\psi_{4}=e^{i \sigma_{0}} C_{\eta}\left\{p^{4}-\left[\frac{4}{3} \alpha M \mu+\frac{5}{6}(\alpha M)^{2}\right] p^{2}+\frac{1}{6}(\alpha M)^{3} \mu+\frac{1}{24}(\alpha M)^{4}\right\} .
$$

Now we calculate loop functions $J_{0}, J_{2}, J_{22}$ and $J_{4}$ in Eqs. (17), (20), (22), (25) by using the results of the $\psi$ functions obtained above.

4. $J_{0}$ :

The function $J_{0}(p)$ is given by [7]

$$
J_{0}(p)=M \int \frac{d^{3} \vec{l}}{(2 \pi)^{3}} \frac{2 \pi \eta(l)}{e^{2 \pi \eta(l)}-1} \frac{1}{p^{2}-l^{2}+i \epsilon},
$$

where $l=|\vec{l}|$. We now separate $J_{0}$ into two parts as [7]

$$
J_{0}(p)=J_{0}^{d i v}+J_{0}^{f i n},
$$

where

$$
\begin{aligned}
J_{0}^{d i v} & =-M \int \frac{d^{3} \vec{l}}{(2 \pi)^{3}} \frac{2 \pi \eta(l)}{e^{2 \pi \eta(l)}-1} \frac{1}{l^{2}} \\
J_{0}^{f i n} & =M \int \frac{d^{3} \vec{l}}{(2 \pi)^{3}} \frac{2 \pi \eta(l)}{e^{2 \pi \eta(l)}-1} \frac{1}{l^{2}} \frac{p^{2}}{p^{2}-l^{2}+i \epsilon} .
\end{aligned}
$$

As $J_{0}^{f i n}$ is already calculated in Ref. [7], by changing the parameter $x=2 \pi \eta(l)$ and using the relation

$$
\int_{0}^{\infty} d x \frac{x}{\left(e^{x}-1\right)\left(x^{2}+a^{2}\right)}=\frac{1}{2}\left[\ln \left(\frac{1}{2 \pi}\right)-\frac{\pi}{a}-\psi\left(\frac{1}{2 \pi}\right)\right],
$$

where $\psi$ is the logarithmic derivative of the $\Gamma$-function, we have

$$
J_{0}^{f i n}=-\frac{\alpha M^{2}}{4 \pi} H(\eta)=-\frac{\alpha M^{2}}{4 \pi} h(\eta)-C_{\eta}^{2} \frac{M}{4 \pi}(i p),
$$

where $\eta=\alpha M /(2 p), H(\eta)=\psi(i \eta)+\frac{1}{2 i \eta}-\ln (i \eta)$, and $h(\eta)=\operatorname{Re} H(\eta)$.

Next we calculate the divergence part $J_{0}^{\text {div }}$ in $d=4-2 \epsilon$ dimension

$$
J_{0}^{d i v}=-M\left(\frac{\mu}{2}\right)^{4-d} \int \frac{d^{d-1} \vec{q}}{(2 \pi)^{d-1}} \frac{2 \pi \eta(q)}{e^{2 \pi \eta(q)}-1} \frac{1}{q^{2}} .
$$

Changing the variable $x=2 \pi \eta(q)=\pi \alpha M / q$, we have

$$
\begin{aligned}
J_{0}^{d i v} & =-M\left(\frac{\mu}{2}\right)^{4-d} \frac{2 \pi^{(d-1) / 2}}{(2 \pi)^{d-1} \Gamma\left(\frac{d-1}{2}\right)}(\alpha \pi M)^{d-3} \int_{0}^{\infty} d x \frac{x^{3-d}}{e^{x}-1} \\
& =-M\left(\frac{\mu}{2}\right)^{4-d} \frac{2 \pi^{(d-1) / 2}}{(2 \pi)^{d-1} \Gamma\left(\frac{d-1}{2}\right)}(\alpha \pi M)^{d-3} \Gamma(4-d) \zeta(4-d),
\end{aligned}
$$


where we have used the relation $\Omega_{d}=2 \pi^{d / 2} / \Gamma(d / 2)$ and $\zeta(z)$ is the Riemann's zeta function. For $d=4-2 \epsilon$ we have

$$
J_{0}^{d i v}=\frac{\alpha M^{2}}{8 \pi}\left[\frac{1}{\epsilon}-3 \gamma+2+\ln \left(\frac{\pi \mu^{2}}{\alpha^{2} M^{2}}\right)\right] .
$$

We also consider the pole for $d=3$, known as the power divergence subtraction (PDS) scheme pole. Using the relation $\lim _{s \rightarrow 1}\left[\zeta(s)-\frac{1}{s-1}\right]=\gamma$, we have the pole at 3 -dimension

$$
J_{0}^{d i v}=-\frac{\mu M}{4 \pi} \frac{1}{3-d}+\cdots,
$$

and thus we include the PDS counter term and have

$$
J_{0}^{\text {div }}=-\frac{M}{4 \pi} \mu+\frac{\alpha M^{2}}{8 \pi}\left[\frac{1}{\epsilon}-3 \gamma+2+\ln \left(\frac{\pi \mu^{2}}{\alpha^{2} M^{2}}\right)\right] .
$$

5. $J_{2}$

$$
J_{2}=\int \frac{d^{3} \vec{q}}{(2 \pi)^{3}} \frac{d^{3} \vec{q}^{\prime}}{(2 \pi)^{3}} \vec{q}^{2}\left\langle\vec{q}^{\prime}\left|\hat{G}_{C}^{(+)}\right| \vec{q}\right\rangle=M \int \frac{d^{3} \vec{q}}{(2 \pi)^{3}} \frac{\psi_{2}(q) \psi_{0}^{*}(q)}{\vec{p}^{2}-\vec{q}^{2}+i \epsilon} .
$$

Using the result of $\psi_{2}$ in Eq. (64), we get

$$
J_{2}(p)=\left[p^{2}-\mu \alpha M-\frac{1}{2}(\alpha M)^{2}\right] J_{0}(p)-\Delta J_{2},
$$

where

$$
\Delta J_{2}=M\left(\frac{\mu}{2}\right)^{4-d} \int \frac{d^{d-1} \vec{k}}{(2 \pi)^{d-1}} \frac{2 \pi \eta(k)}{e^{2 \pi \eta(k)}-1}=M\left(\frac{\mu}{2}\right)^{4-d} \frac{\Omega_{d-1}}{(2 \pi)^{d-1}}(\pi \alpha M)^{d-1} \Gamma(2-d) \zeta(2-d) .(82
$$

For $d=4$ we have

$$
\Delta J_{2}=\frac{1}{4} \pi \alpha^{3} M^{4} \zeta^{\prime}(-2),
$$

where $\zeta^{\prime}(-2)=-0.0304 \cdots$. For $d=3$ we obtain

$$
\Delta J_{2}^{(d=3)}=\frac{1}{48} \pi \alpha^{2} M^{3} \mu \frac{1}{3-d}+\cdots,
$$

and by including the PDS counter term we have

$$
\Delta J_{2}=\frac{\pi M}{48}(\alpha M)^{2} \mu+\frac{\pi M}{4}(\alpha M)^{3} \zeta^{\prime}(-2) .
$$

6. $J_{22}$

$$
\begin{aligned}
J_{22} & =\int \frac{d^{3} \vec{q}}{(2 \pi)^{3}} \frac{d^{3} \vec{q}^{\prime}}{(2 \pi)^{3}} \vec{q}^{2}\left\langle\vec{q}^{\prime}\left|\hat{G}_{C}^{(+)}\right| \vec{q}\right\rangle \vec{q}^{2}=M \int \frac{d^{3} \vec{q}}{(2 \pi)^{3}} \frac{\psi_{2}(q) \psi_{2}^{*}(q)}{p^{2}-q^{2}+i \epsilon} \\
& =\left(p^{4}-2 A p^{2}+A^{2}\right) J_{0}-\left(p^{2}-2 A\right) \Delta J_{2}-\Delta J_{22},
\end{aligned}
$$


where $A=\mu \alpha M+\frac{1}{2}(\alpha M)^{2}$, and

$$
\begin{aligned}
\Delta J_{22} & =M\left(\frac{\mu}{2}\right)^{4-d} \int \frac{d^{d-1} \vec{q}}{(2 \pi)^{d-1}} \vec{q}^{2} \psi_{0}(q) \psi_{0}^{*}(q) \\
& =M\left(\frac{\mu}{2}\right)^{4-d} \frac{\Omega_{d-1}}{(2 \pi)^{d-1}}(\pi \alpha M)^{d+1} \Gamma(-d) \zeta(-d) .
\end{aligned}
$$

For $d=4$ we have

$$
\Delta J_{22}=\frac{1}{48} \pi^{3} \alpha^{5} M^{6} \zeta^{\prime}(-4),
$$

where $\zeta^{\prime}(-4)=0.00798 \cdots$ For $d=3$ we get

$$
\Delta J_{22}^{(d=3)}=-\frac{1}{2880} \pi^{3} \alpha^{4} M^{5} \mu \frac{1}{3-d}+\cdots,
$$

and thus we obtain

$$
\Delta J_{22}=-\frac{\pi^{3} M}{2880}(\alpha M)^{4} \mu+\frac{\pi^{3} M}{48}(\alpha M)^{5} \zeta^{\prime}(-4) .
$$

7. $J_{4}$

$$
J_{4}=\int \frac{d^{3} \vec{q}}{(2 \pi)^{3}} \frac{d^{3} \vec{q}^{\prime}}{(2 \pi)^{3}} \vec{q}^{4}\left\langle\vec{q}^{\prime}\left|\hat{G}_{C}^{(+)}\right| \vec{q}\right\rangle=M \int \frac{d^{3} \vec{q}}{(2 \pi)^{3}} \frac{\psi_{4}(q) \psi_{0}^{*}(q)}{p^{2}-q^{2}+i \epsilon}
$$

Using the relation for $\psi_{4}$ in Eq. (68), we have

$$
\begin{aligned}
J_{4}= & \left\{p^{4}-\left[\frac{4}{3} \alpha M \mu+\frac{5}{6}(\alpha M)^{2}\right] p^{2}+\frac{1}{6}(\alpha M)^{3} \mu+\frac{1}{24}(\alpha M)^{4}\right\} J_{0} \\
& -\left[p^{2}-\frac{4}{3} \alpha M \mu-\frac{5}{6}(\alpha M)^{2}\right] \Delta J_{2}-\Delta J_{22} .
\end{aligned}
$$

\section{References}

[1] S. R. Beane et al., in At the Frontier of particle Physics, edited by M. Shifman (World Scientific, Singapore, 2001) Vol. 1, p. 133; nucl-th/0008064.

[2] P. F. Bedaque and U. van Kolck, Annu. Rev. Nucl. Part. Sci. 52 (2002) 339.

[3] K. Kubodera and T.-S. Park, Annu. Rev. Nucl. Part. Sci. 54 (2004) 19.

[4] E. Epelbaum, Prog. Part. Nucl. Phys. 57 (2006) 654.

[5] V. Bernard, arXiv:0706.0312. 
[6] X. Kong and F. Ravndal, Phys. Lett B 450 (1999) 320.

[7] X. Kong and F. Ravndal, Nucl. Phys. A665 (2000) 137.

[8] D. B. Kaplan, M. J. Savage, M. B. Wise, Phys. Lett. B 424 (1998) 390.

[9] D. B. Kaplan, M. J. Savage, M. B. Wise, Nucl. Phys. B534 (1998) 329.

[10] R. B. Wiringa, V. G. J. Stoks, R. Schiavilla, Phys. Rev. C 51 (1995) 31.

[11] I. Šlaus, Y. Akaishi and H. Tanaka, Phys. Rep. 173 (1989) 257.

[12] G. A. Miller, B. M. K. Nefkens, and I. Šlaus, Phys. Rep. 194 (1990) 1.

[13] C. R. Howell et al., Phys. Lett. B 444 (1998) 252.

[14] D. E. González Trotter et al., Phys. Rev. Lett. 83 (1999) 3788.

[15] V. Huhn et al., Phys. Rev. Lett. 85 (2000) 1190.

[16] W. von Witsch, X. Ruan and H. Witala, Phys. Rev. C 74 (2006) 014001.

[17] A. Gardestig and D. R. Phillips, Phys. Rev. C 73 (2006) 014002.

[18] S. Ando and K. Kubodera, Phys. Lett. B 633 (2006) 253.

[19] S. Albeverio et al., Phys. Rev. C 29 (1984) 680.

[20] R. Machleidt, Phys. Rev. C 63 (2001) 024001.

[21] P. U. Sauer and H. Walliser, J. Phys. G 3 (1977) 1513; M. Rahman and G. A. Miller, Phys. Rev. C 27 (1983) 917.

[22] M. Walzl, U.-G. Meißner, and E. Epelbaum, Nucl. Phys. A693 (2001) 663.

[23] J.-W. Chen, G. Rupak, and M. J. Savage, Nucl. Phys. A653 (1999) 386; M. Butler and J.-W. Chen, Phys. Lett. B 520 (2001) 87; M. Butler, J.-W. Chen, and X. Kong, Phys. Rev. C 63 (2001) 035501.

[24] See, e.g., Charles J. Joachain, Quantum Collision Theory, North-Holland (1975).

[25] H. A. Bethe, Phys. Rev. 76 (1949) 38.

[26] S. Fleming, T. Mehen, and I. W. Stewart, Nucl. Phys. A677 (2000) 313.

[27] L. Durand, III, Phys. Rev. 108 (1957) 1597; L. Heller, Phys. Rev. 120 (1960) 627.

[28] D. Binosi and L. Theussl, Computer Physics Communications 161 (2004) 76.

[29] J. Gegelia, nucl-th/9802038. 
[30] J. Gasser, A. Rusetsky, and I. Scimemi, Eur. Phys. J. C 32 (2003) 97.

[31] J. Gegelia, Eur. Phys. J. A 19 (2004) 355.

[32] B. Podolsky and L. Pauling, Phys. Rev. 34 (1929) 109. 